\title{
Evolution of anodic stress corrosion cracking in a coated material
}

\author{
C. Bjerkén - M. Ortiz
}

Received: 11 October 2009 / Accepted: 3 June 2010 / Published online: 25 June 2010

(C) The Author(s) 2010. This article is published with open access at Springerlink.com

\begin{abstract}
In the present paper, we investigate the influence of corrosion driving forces and interfacial toughness for a coated material subjected to mechanical loading. If the protective coating is cracked, the substrate material may become exposed to a corrosive media. For a stress corrosion sensitive substrate material, this may lead to detrimental crack growth. A crack is assumed to grow by anodic dissolution, inherently leading to a blunt crack tip. The evolution of the crack surface is modelled as a moving boundary problem using an adaptive finite element method. The rate of dissolution along the crack surface in the substrate is assumed to be proportional to the chemical potential, which is function of the local surface energy density and elastic strain energy density. The surface energy tends to flatten the surface, whereas the strain energy due to stress concentration promotes material dissolution. The influence of the interface energy density parameter for the solid-fluid combination, interface corrosion resistance and stiffness ratios between coating and sub-
\end{abstract}

\section{Bjerkén (ه)}

Division of Materials Science, School of Technology,

Malmö University, 20506 Malmö, Sweden

e-mail: christina.bjerken@mah.se

C. Bjerkén

Division of Materials Engineering, Lund Institute of Technology, PO Box 118, 22100 Lund, Sweden

\section{Ortiz}

Department of Engineering and Applied Science, California Institute of Technology, 1200 East California Boulevard, Mail code: 105-50, Pasadena, CA 91125, USA strate is investigated. Three characteristic crack shapes are obtained; deepening and narrowing single cracks, branched cracks and sharp interface cracks. The crack shapes obtained by our simulations are similar to real sub-coating cracks reported in the literature.

Keywords Stress corrosion - Layered material . Moving boundary problem - Surface energy density . Strain energy density $\cdot$ Interface toughness

\section{Introduction}

Coatings are often used to improve the corrosive, thermal, tribological, electrical and mechanical properties of metals, ceramics and polymers. Under the presence of residual stresses, static and fatigue loads, the protective coating may develop cracks thus exposing the substrate material to a potentially aggressive environment. Consequently, the combined effect of mechanical loads and a corrosive environment may lead to the development of stress corrosion cracks in the base material.

The mechanisms of stress corrosion cracking in metals can be classified as (i) anodic mechanisms, e.g. active dissolution and removal of material from the crack tip; and (ii) cathodic mechanisms, e.g. hydrogen evolution, adsorption, diffusion and embrittlement. In this study, we focus on the former group, particularly on the study of stress-driven material dissolution where the loss of atoms to the environment may lead to crack growth. The interaction of the dissolution 


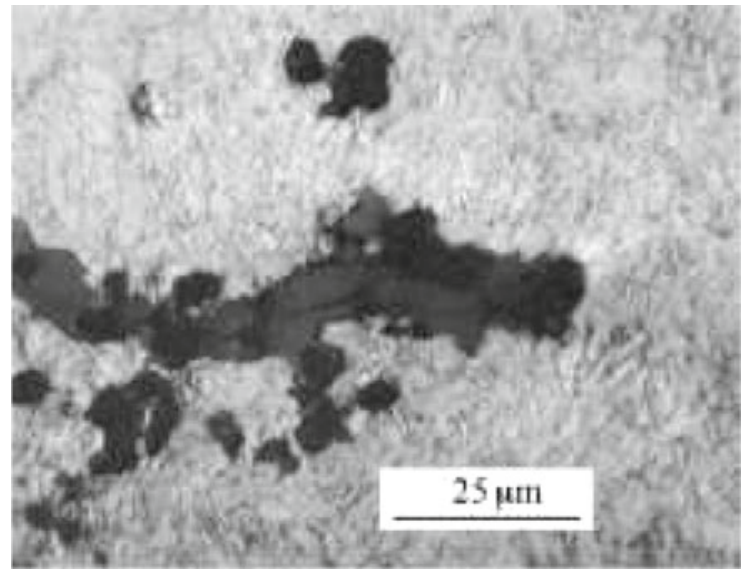

Fig. 1 Corrosion crack in a pressure vessel steel of type SA5331C11. Crack length is around $7 \mathrm{~mm}$ and notch width around $10 \mu \mathrm{m}$. Reproduced with permission from Vattenfall AB, Sweden

process and the mechanical loads may render local irregularities that develop into pits and notches and eventually into cracks. Usually, these cracks do not show any distinct borderline and are, therefore, integral parts of the body surface. The dissolution process defines the growth rate and growth direction. Figure 1 shows the crack tip region of a stress corrosion crack on steel belonging to a nuclear pressure vessel. The observed gap is the area where the material has been dissolved through an anodic corrosion process. It can be noticed that the crack tip has a blunted shape as long as it grows through material dissolution, which is supported by i.e. Ståhle et al. (2007).

Much attention has been given to stress-induced roughening of solid surfaces. A stressed flat surface is unstable, and when mass transportation such as dissolution or surface diffusion is present, the mechanical stress has been found to produce a surface waviness. The phenomenon is theoretically explained by Asaro and Tiller (1972), Grinfeld (1986) and Srolovitz (1989). Mass transportation through etching is considered by Kim et al. (1999). The wave spectrum of the developing surface roughness depends on the stress in the body surface and the surface energy. The theory is based on the recognition of the surface energy and the elastic strain energy providing driving forces for material dissolution. A large surface energy diminishes the waviness and a large elastic strain energy amplifies it. A critical wavelength, $\lambda_{c r}$, can be identified for a surface with a shallow waviness. The result is that the amplitude of waves with wavelengths longer than this $\lambda_{c r}$ increases, while waves with shorter wavelengths is suppressed with time.

Sopok et al. (2005a,b) and Underwood et al. (2004, 2007) reported material removal and crack development at the interface between coating and substrate material in gun barrels. Even though their studies focussed on the wear and cracking of the coating inside a gun bore, they also reported cracking in the substrate material. This coating-substrate system was subjected to both thermal and mechanical loading as well as wear and erosion in an aggressive environment. Figure 2 shows schematic illustrations of different crack patterns from micrographs in Sopok et al. (2005a,b), Underwood et al. (2004, 2007). The attacks are found at locations where cracks in the coating reach the interface. As the coating cracks are opened during loading, the aggressive environment is assumed to encounter the more sensible substrate. It can also be seen in Fig. 2 that both the dissolution of the substrate material along the interface and pit shaped corrosion attacks seem plausible. Some of the deep pits have probably developed into sharp cracks.

\section{Problem formulation}

In the present study, an initial corrosion attack of the substrate is introduced in the form of a small halfcircular pit, see Fig. 3. The influence of thermal gradients in the referred real system is disregarded, and only mechanical loading applied parallel to the interface is considered. Both the coating and the substrate are assumed to be isotropic, linear elastic solids with Young's modulus $E_{c}$ and $E_{s}$, and Poisson's ratio $v_{c}$ and $v_{s}$, respectively, where the subscript $c$ refers to the coating and $s$ to the substrate. Here, $v_{c}=v_{s}$ and set equal to 0.3. Three different material combinations are studied; a weaker coating, a homogeneous material and a stiffer coating with the stiffness ratio $E_{c} / E_{s}=0.1,1$ and 10, respectively. As an additional information, the corresponding Dundurs' parameters (Dundurs 1969) are given: $\alpha_{D} \approx[-0.82,1,0.82]$ and $\beta_{D} \approx[-0.23,1,0.23]$.

In this investigation, a model with a finite geometry and boundary conditions as shown in Fig. 4 is considered. A two-dimensional Cartesian coordinate system is introduced, with its origin at the tip of the crack in the coating. Due to the symmetry about the $x$ axis, only 
Fig. 2 Rough sketches of micrographs of findings reported in (Sopok et al. 2005a,b; Underwood et al. 2004, 2007)
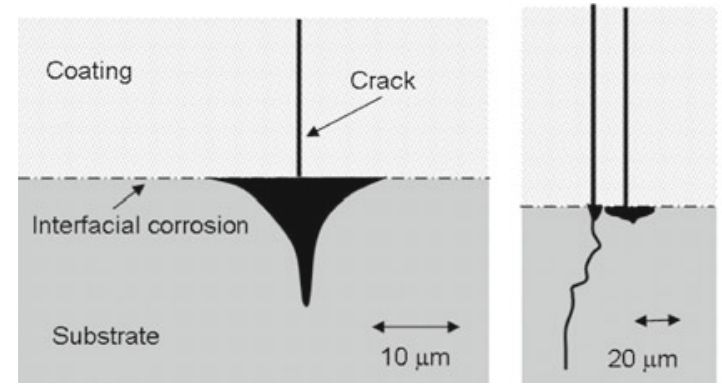

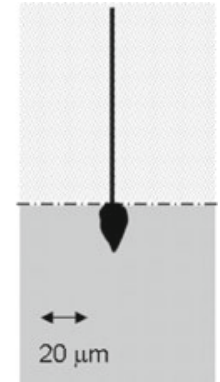

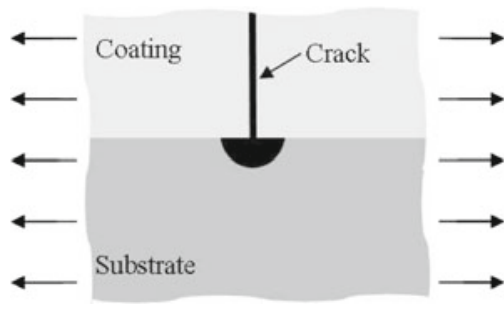

Fig. 3 Initial pit shaped as a half-circle. The direction of the applied load is illustrated with arrows

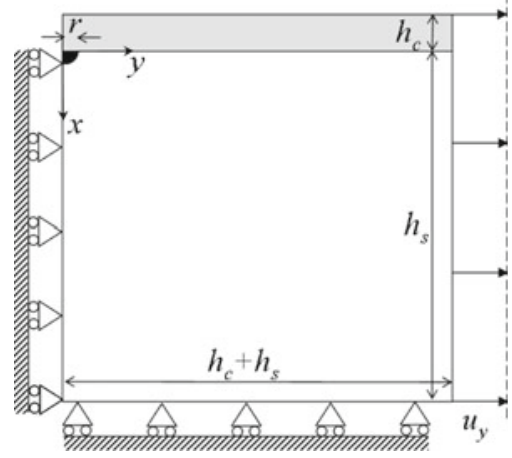

Fig. 4 Geometry and boundary conditions of the model

one half of the geometry is need for the analysis. The thickness of the coating is denoted $h_{c}$, the substrate thickness $h_{s}$ and is chosen equal to $9 h_{c}$, and the width equals $h_{c}+h_{s}$. The initial pit radius $r=10^{-2} h_{c}$. A constant displacement, $u_{y}$, is applied at the boundary at $y=h_{c}+h_{s}$ for $-h_{c}<x<h_{s}$, and results in a nominal strain in the body $\varepsilon_{0}=u_{y} /\left(h_{c}+h_{s}\right)$ The bottom of the substrate $\left(x=h_{s}\right)$ is restricted from moving in the $x$ direction, and due to the symmetry, the substrate at $y=0$ is not allowed to move in the $y$ direction. The remaining boundaries are assumed to be free of tractions.

\subsection{Surface evolution}

The evolution of the surface is assumed to result from corrosion where the material is dissolved into a surrounding aggressive media, see Fig. 5. This media acts as an infinite buffer, i.e. the evolution is not influenced by concentration changes in the liquid. The chemical potential, $\chi$, of a reaction at a given location is governed by the amount of energy density available there, and in the case of corrosion also of the electro-chemical potential for the system. For a stressed material, the elastic strain energy density $U_{\varepsilon}$ gives one contribution to the energy. Another contribution is $U_{\gamma}$, which represents the local surface energy density that varies with the surface curvature. The tendency for a material to change the shape of its reference configuration is represented by the chemical potential, cf. Kim et al. (1999):

$\chi=\Omega\left(g_{0}+U_{\gamma}+U_{\varepsilon}\right)$,

with $\Omega$ being the atomic volume, and $g_{0}$ is the electro-chemical potential of the corrosion reaction, which here is assumed to be a constant since the material is homogeneous and the corrosive medium is an infinite buffer. The electro-chemical potential may promote or hold back the corrosion reactions depending on sign and magnitude. For a nearly flat surface, a non-zero $g_{0}$ causes a translation of the surface. For the sub-surface cracks, $U_{\gamma}$ and $U_{\varepsilon}$ are here assumed to dominate over $g_{0}$ due to the relatively large stresses and curvature associated with the crack growth.

The rate of evolution at locations along the surface, $v(s)$, due to stress corrosion is assumed to be proportional to $\chi(s)$, where $s$ is a curvilinear coordinate following the surface. Disregarding the constant $g_{0}$, Eq. (1) gives the magnitude of dissolution normal to the surface at $s$. Therefore, the evolution rate can be written as:

$v(s)=C\left(U_{\gamma}(s)+U_{\varepsilon}(s)\right)$, 


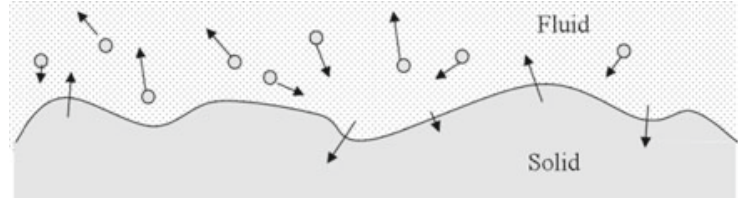

Fig. 5 Corroding surface of a solid in contact with a corrosive fluid

where $C$ is a constant. Furthermore, $U_{\gamma}$ is assumed to vary with surface curvature, $\kappa(s)$, as

$U_{\gamma}(s)=\kappa(s) \gamma$,

with $\gamma$ denoting the interface energy density of the solid-fluid interface and is a constant for the considered system. The curvature $\kappa$ is defined positive at locations where the surface forms hills, and negative at valleys. With this definition of $\kappa$, it can be concluded that $U_{\gamma}$ will hold back the dissolution in valleys, and vice versa. From a physical point of view, this reflects the fact that atoms are more loosely bonded due to fewer neighbouring atoms at hills and thus more easily dissolved into the surrounding. On the contrary, at the bottom of a valley atoms are more firmly bonded.

Now, consider a body with a homogeneous and isotropic, linear elastic material with Young's modulus $E$ and Poisson's ratio $v$. It is assumed to always be in mechanical equilibrium under plane stress or strain. The strain energy density along the surface, without any tractions acting on it, can then be expressed as

$U_{\varepsilon}(s)=\bar{E} \varepsilon(s)^{2} / 2$,

where $\varepsilon(s)$ is the surface strain, and $\bar{E}=E$ for plane stress and $\bar{E}=E /\left(1-v^{2}\right)$ for plane strain. $U_{\varepsilon}$ will increase dissolution at in-going parts of the surface, which act as stress concentrators.

Insertion of Eqs. (3) and (4) into (2), gives the governing equation for surface evolution used in the numerical method:

$v(s)=C\left(\bar{E} \varepsilon(s)^{2}+\kappa(s) \gamma\right)$.

In the following, the constant $C$ is set equal to unity. As mentioned in the Introduction, a flat stressed surface is unstable. With mass transportation mechanisms such as dissolution, deposition or diffusion, the competition between $U_{\gamma}$ and $U_{\varepsilon}$ leads to a roughening with a characteristic wave spectrum. With a linearized theory assuming that the amplitude of the height variation of the body surface is small, it leads to symmetric growth in the sense that the growth rate of hills, as an average, is the same as at valleys, and a critical wavelength can be found, cf. (Kim et al. 1999; Asaro and Tiller 1972):

$\lambda_{c r}=\frac{\pi \gamma}{\varepsilon_{0}^{2} \bar{E}}$,

where $\varepsilon_{0}$ is the nominal strain of the corresponding flat surface. Wavelengths shorter than $\lambda_{c r}$ will decay, and there also is a dominating wavelength equal to $\lambda_{\max }=$ $2 \lambda_{c r}$ for which the amplitude will increase the fastest.

\section{Numerical method}

The numerical method is an adaptive procedure and is a modification of a method earlier developed by Jivkov and Ståhle (2002). They studied corrosion fatigue due to repeated film rupture using a different surface evolution law than that used herein. The method is based on calculations of surface strains using the finite element (FE) code ABAQUS (2007). The curvature along the pit surface is determined at each node of the FE mesh. The evolution of the surface is then found by adopting Eq. (5). Jivkov and Ståhle (2002) instead assumed that the dissolution rate was a linear function of the strain along the crack surface.

For the FE analysis, 3-node constant strain elements are used. The FE mesh is generated by a Delaunytype triangulation using the freeware code Triangle, cf. Shewchuk (2002). In Fig. 6, the mesh of the initial geometry is shown both for the overall geometry and near the pit.

At the node $i$ at the pit surface, the strain $\varepsilon_{i}$ is obtained as the mean value of the strains of the two adjacent element sides at the surface.

The surface curvature $\kappa$ in each node is found using a discrete version of

$\kappa(s)=\frac{x^{\prime \prime}(s) y^{\prime}(s)-x^{\prime}(s) y^{\prime \prime}(s)}{\left(x^{\prime}(s)^{2}+y^{\prime}(s)^{2}\right)^{3 / 2}}$,

where ' denotes the first derivative and " the second.

The corrosion process is simulated as a surface evolution that advances stepwise for small time increments $t_{n}$. The step in each node $l_{i}$ equals $v_{i} t_{n}$, where $v_{i}$ is the dissolution rate in the normal direction of the surface in node $i$. The rate $v_{i}$ is assumed to be constant during the time increment. To ensure that the incremental evolution of the surface is not accelerating too much due to developing stress concentrations eventually resulting in numerical problems, a maximum corrosion depth 
Fig. 6 a Meshed geometry b Close-up at the pit region. The shaded area represents the coating (a)

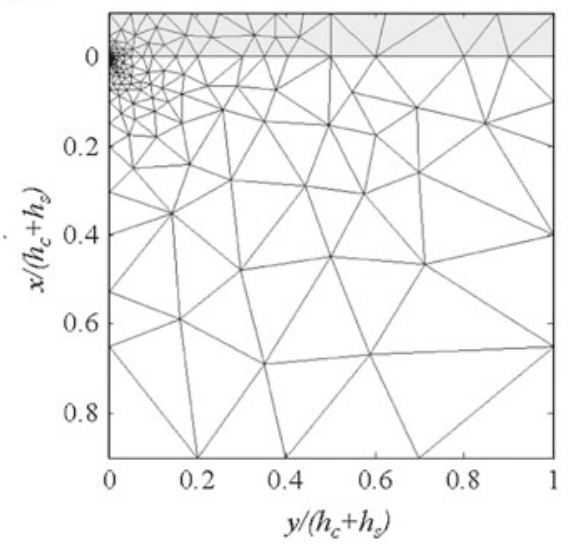

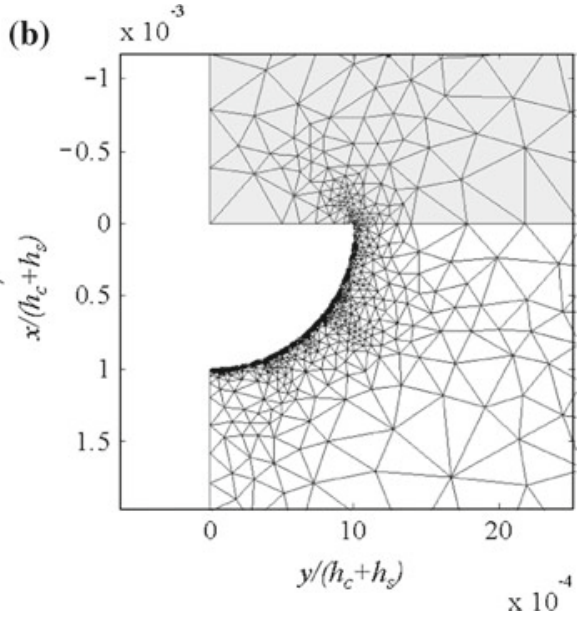

$l_{\max }$ governs the size of the time step, $t_{i}$, in each computational step. We assume that the main features of the surface evolution can be captured by this procedure using sufficiently small time increments.

For each time increment, the FE element analysis is performed. Thereafter the evolution rate in each node is computed, and a new geometry is determined. Due to the surface evolution, the distance between nodes along the corroding surface changes. Thus, a routine is adopted where nodes are added or removed if a maximum or minimum distance between nodes, respectively, is exceeded. Before the next iteration the new geometry is re-meshed.

If the growth rate solely depends on the surface strains, the evolution is inherently sensitive to perturbations arising from e.g. the FE mesh, see e.g. Jivkov (2004) and Bjerkén (2010). Local depressions in the surface act as stress raisers may render a wobbling growth path and initiation of branching. In order to reduce this effect, a cubic B-spline curve can be created along the new surface in each fatigue cycle, cf. (Ståhle et al. 2007). With the evolution law adopted here, the $U_{\gamma}$ and $U_{\varepsilon}$ will counteract each other and thus render a more stable surface evolution per se. However, to ensure that the influence of local variations along the surface arising due to the discretized geometry is small, we use a regular low pass filter.

\section{Results and discussion}

The interface energy parameter $\gamma$ governs the influence of the curvature on the evolution rate, cf. Eq. (5).
This study investigates the influence of different values of $\gamma$ especially on the evolving shape of a stress corrosion crack which origins at the interface between two different materials. The interface toughness strongly affects the surface evolution along a bi-material interface. It can be easily reasoned that a weak interface will facilitate the spreading of the corrosion attack along the interface. The interface toughness is modelled by using different geometric restrictions for surface evolution in a small region close to the interface. We also investigate the influence of different stiffness ratios between coating and substrate.

A reference value $\gamma_{0}$ is introduced:

$\gamma_{0}=\frac{100 \lambda_{\max }}{\pi} U_{\varepsilon 0}$,

where $\lambda_{\max }$ is the dominating wavelength in the case of a nearly flat surface with $\gamma=100 \gamma_{0}$ and a strain energy density $U_{\varepsilon 0}$ along the surface. In the present study, $U_{\varepsilon 0}$ is defined as a nominal strain energy density:

$U_{\varepsilon 0}=\bar{E}_{s} \varepsilon_{0}^{2} / 2$.

Here, $\lambda_{\max }$ is set equal to the pit radius, $r$. The simulations are performed for 100 increments in most cases, and a few broke earlier due to numerical difficulties.

\subsection{Influence of interface toughness}

The initial pit meets the interface in a $90^{\circ}$ corner. A wedge, like this, in a bi-material acts as a strong stress concentrator. For a linear elastic material, the stresses are singular at the interface. In this study, the singular 
Fig. 7 Adjustment of node locations within a near interface zone according to different interface toughness. a Rule I (medium), b Rule II (weak), and c Rule III (tough)

Fig. 8 Influence of interface toughness on shape evolution with $\gamma=\gamma_{0}$ : a Rule I (medium) b Rule II (weak), and c Rule III (tough). Several steps in the simulations are left out for clarity (a)

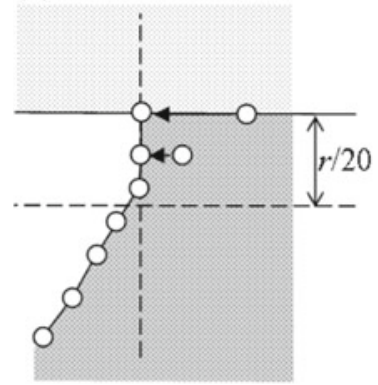

(a)

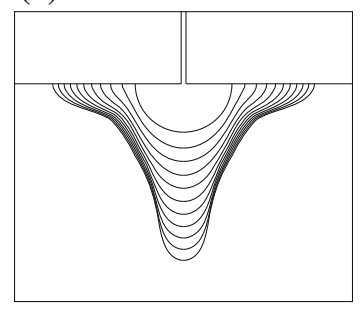

(b)

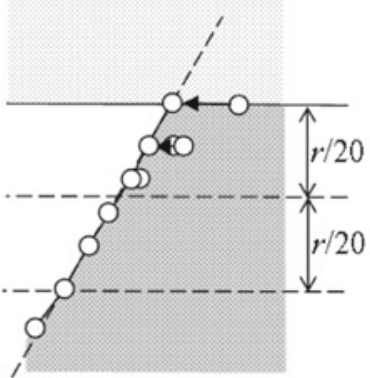

(b)

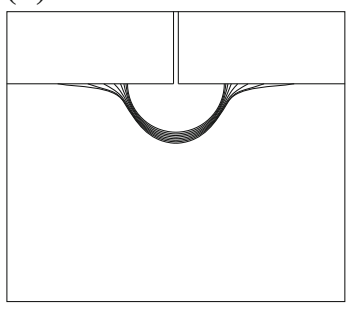

(c)

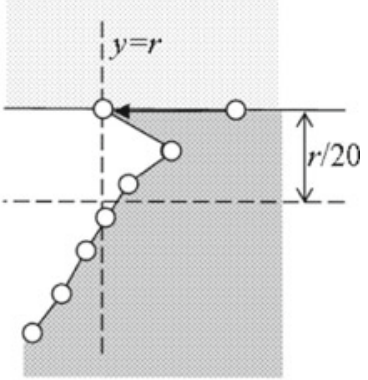

(c)

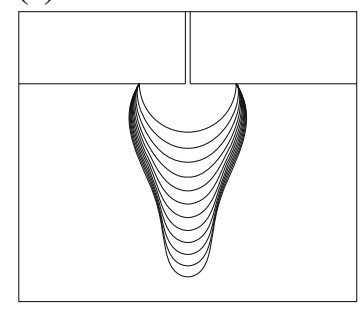

stresses are not taken into account, and, thus, a fracture criterion is not included. Instead, the influence of different interface toughness is dealt with in a simplified way. Three types of geometric constraints are adopted for the surface evolution near the interface between the coating and the substrate. As new node positions are determined according to the evolution law (Eq. 5), the nodes closest to the interface (in a zone where $0<x<r / 20$ ) are adjusted according to three simple rules. Rule I states that all the nodes within the region must have the same $y$ coordinate as the node closest to $x=r / 20$, see Fig. 7a. With the next rule, II, the nodes in the interval $r / 20<x<r / 10$ are used for a linear extrapolation to find the new node coordinates (Fig. 7b). Rule III says that the node located at the interface is kept at its original location at $(x, y)=(0, r)$ throughout the simulation, i.e. is not moved (Fig. 7c). These three rules can be thought of as different interface resistance to stress corrosion. Rule II represents the weakest interface, Rule III the toughest one, whereas Rule I represents a medium resistance.

The different types of resistance of the near-interface region might have a physical relevance. Large toughness could be a result of different material properties in this region due to a mix of substrate and coating species, like, for example, higher chromium content compared to the bulk material. The weak interface rule, II, could be interpreted as a pre-cracked interface with its acccompanying large stress concentration.
The influence of these rules on the surface evolution is investigated. Simulations are performed for the case with stiffer coating $\left(E_{c} / E_{s}=10\right)$ and with $\gamma=\gamma_{0}$. Results are presented in Fig. 8 in the form of contours for every 10 th increment. The weak interface (Rule II) is found to render sharp interface cracks, with only an initial deepening of the pit. On the other hand, branching is found to occur for the medium resistance case. Two symmetric branches follow the interface, whereas one grows perpendicular into substrate. All branches have blunt tips. Finally, for a tough interface, the initial pit is found to deepen and get narrower while keeping a blunt tip.

As said above, the time increments in each simulation are adjusted so that the node with the largest evolution rate is moved a fixed step. This means that the evolution contours in Fig. 8 do not reflect the evolution with time. Figure 9 shows the evolution rates of the node at the deepest point of the pit and a node close to the interface for the three cases in Fig. 8. The growth rate $v$ is normalised with the initial growth rate, $v_{0}$ at the pit bottom (tip) for the medium tough interface. A reference time $t_{0}$ is chosen corresponding to the 50th step in the same simulation. With this medium tough interface, the tip moves approximately with a constant speed. The evolution rate of the tip of an interface branch is slowly decreasing. With a tough interface, the growth rate at the tip is approximately constant, and near the interface very close to zero, which is expected since the 


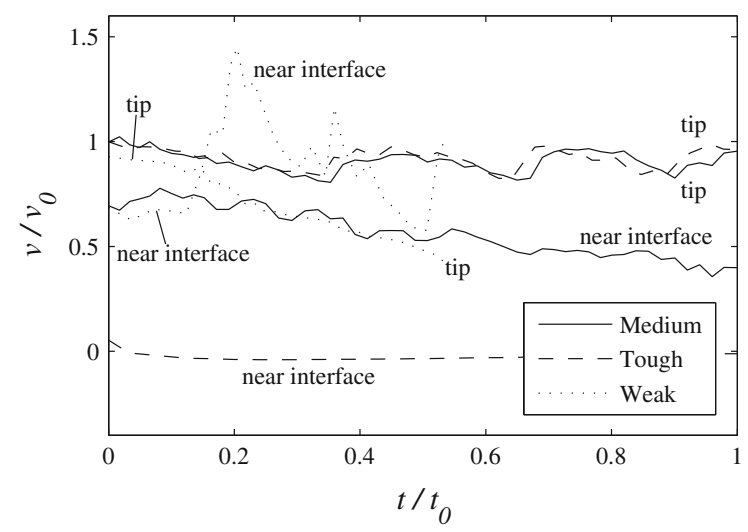

Fig. 9 Growth rate, $v$, during evolution at the bottom (tip) of the pit and near the interface for the three different types of interface toughness

node at the very interface is fixed. In the case with a weak interface, the bottom of the pit initially grows somewhat faster than near the interface and then slows down. As the interface crack develops, it will sharpen and the growth rate increases. Finally, the growth rate vary substantially since the numerical method can no longer follow the development properly, due to the large stress concentration at the interface crack tip, and the simulation is stopped.

\subsection{Influence of surface energy}

The influence of the surface energy density is studied for the case with a stiffer coating, $E_{c} / E_{s}=10$. The parameter $\gamma$ is varied between $10^{-2} \gamma_{0}$ and $10^{3} \gamma_{0}$, where low values of it result in strain energy dominated evolution. Figure 10 shows the shape evolution for simulations with $\gamma / \gamma_{0}$ equal to $10^{-2}, 1$, and $10^{2}$, respectively, for an interface with medium toughness. The characteristic branched shape develops for $\gamma \leq 10 \gamma_{0}$, cf. Fig. 10a and b. Blunted cracks are growing both along the interface and perpendicular to it. For higher

Fig. 10 Influence of surface energy density on shape evolution with medium interface resistance (Type I): a $\gamma / \gamma_{0}=10^{-2}$, b $\gamma / \gamma_{0}=1$, and $\mathbf{c}$ $\gamma / \gamma_{0}=10^{2}$

$\gamma$ values, the stress corrosion attack is held back and will not develop into crack-like shapes. Its only effect is to decrease the pit radius or to give a slow extension into the substrate, cf. Fig. 10c. With a weak interface (Rule II), most pits are found to evolve into interface cracks as in Fig. 8b. Only for $\gamma=10^{3}$, the surface energy density is large enough to hinder growth along the interface. For tough interfaces (Rule III), the evolution follows the same patterns as shown in Fig. 8c.

\subsection{Influence of coating stiffness}

Additionally, some simulations are performed to compare the influence of stiffness ratio between the coating and substrate. Three different situations are investigated: A homogeneous material with $E_{c} / E_{s}=1$, a stiffer coating with $E_{c} / E_{s}=10$, and a weaker with $E_{c} / E_{s}=1 / 10$.

The strain distribution along the pit is determined by the external load, geometry and the material properties. For the investigations used in the Sects. 4.1 and 4.2, we have used a coating that is 10 times stiffer than the substrate material, i.e. $E_{c} / E_{s}=10$. Now, we consider the case where $E_{c} / E_{s}=1$, i.e. where coating and the substrate material have the same material properties except for the corrosion resistance. The coating is still considered immune. To compare, some simulations with this, here called, homogenous material are performed for different interface toughness, where $\gamma=\gamma_{0}$. Figure 11 shows the surface evolution for the case with a weak interface. No tendency to develop sharp cracks at the wedges is found during the 25 increments applied. This is expected, since it is not a bi-material wedge, it will not act as a strong stress raiser. Figure 12 shows the distribution of energy density, $U_{\varepsilon}, U_{\gamma}$ and the sum $U_{\text {tot }}$, along the pit surface for the last time increment. For the two tougher types of interface the same evolution pattern is obtained, which also is expected.

(b)

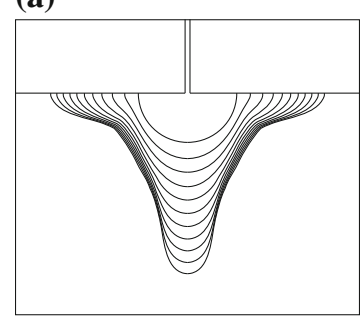

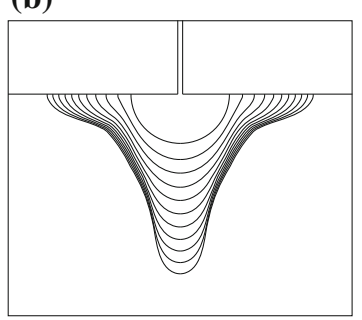

(c)

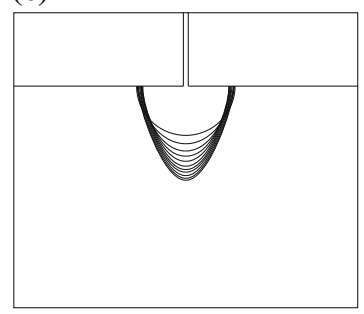




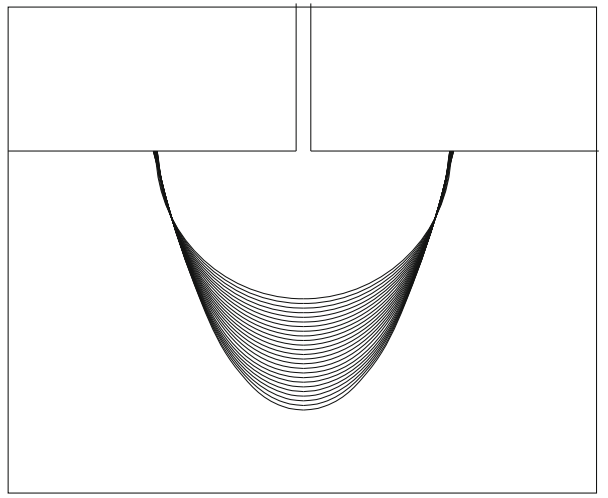

Fig. 11 Surface evolution of the pit for the homogeneous material $\left(E_{c} / E_{s}=1\right)$ with $\gamma=\gamma_{0}$. The evolution is similar for all three types of interface toughness

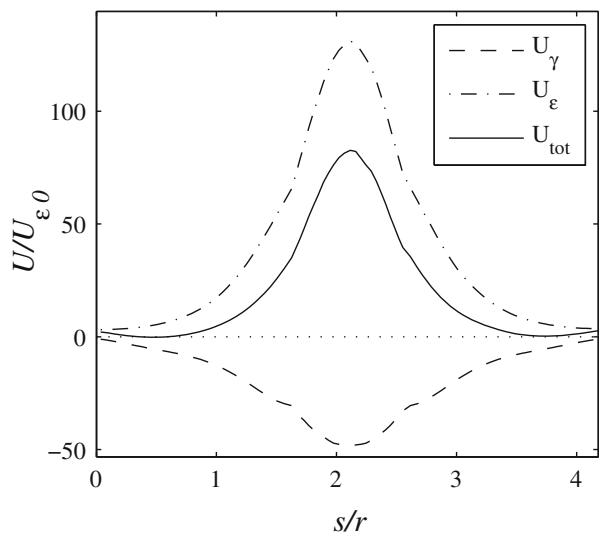

Fig. 12 Energy densities along the pit surface for the last iteration shown in Fig. 11

A substrate coated with a weaker material is also considered. Here, the surface evolution for $E_{c} / E_{s}=$ 0.1 is simulated. With a medium tough interface, the pit is found to shrink for $\gamma \geq \gamma_{0}$, and form branches for $\gamma \leq 0.01 \gamma_{0}$. For $\gamma=0.1 \gamma_{0}$ an V-shaped equilibrium shape evolves, see Sect. 4.4 below. Also with weaker coating, the stresses are concentrated at the interface, meaning that branching or interface cracking may develop also in this case. Figure 13 shows the strain energy density distribution along the surface of the initial half-circular pit for the three stiffness ratios considered. In all cases, the largest $U_{\varepsilon}$ is found at the bottom of the pit. As the interface is approached it declines but for the two bimaterials it increases again when getting close to the wedge. With a weaker coating $\left(E_{c} / E_{s}=0.1\right)$, the strain energy density drops relatively more before increasing at the interface than with the stiff one.

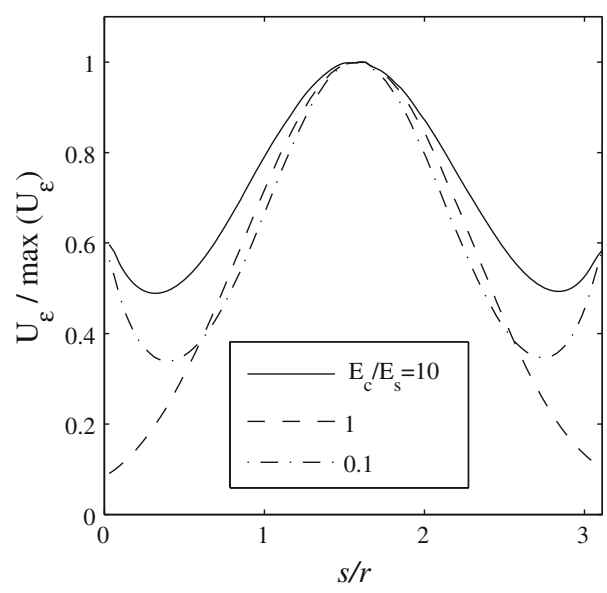

Fig. 13 Distribution of strain energy density, $U_{\varepsilon}$, along pit surface of the initial half-circular pit for different stiffness ratios, $E_{c} / E_{s}$, normalised with the maximum strain energy density in each case

\subsection{Weaker coating and equilibrium shape}

With a weaker coating $\left(E_{c} / E_{s}=0.1\right)$, an equilibrium shape was achieved for $\gamma=0.1 \gamma_{0}$. Figure 14 shows the evolution during 30 time increments. The pit is found to evolve slowly into a V-shaped notch with a blunt tip. The opening angle of the notch is approximately equal $70^{\circ}$. After approximately 25 iteration steps, the evolution stops in the tip region, and near the interface small fluctuations are found, where the surface repeatedly moves outwards and inwards. The growth rate during the evolution for the tip and near the interface is presented in Fig. 15. Note that both growth rate and time are normalised with the same reference values $v_{0}$ and $t_{0}$ as in Fig. 9. This means, that the growth is very slow for a long time, which supports the idea that an equilibrium state is reached. Of course, for an equilibrium to occur the two kinds of energy densities must be equal but with different signs along the whole surface. Figure 16 shows the distribution of $U_{\varepsilon}, U_{\gamma}$ and the sum $U_{\text {tot }}$ for the 25 th increment, and the balance appears to be very good. The small deviation near the interface changes from positive to negative and gives the oscillation of the surface there.

\subsection{Maximum strain energy density}

In absolute terms, the maximum strain energy density that is found at the bottom of the initial pit increases 


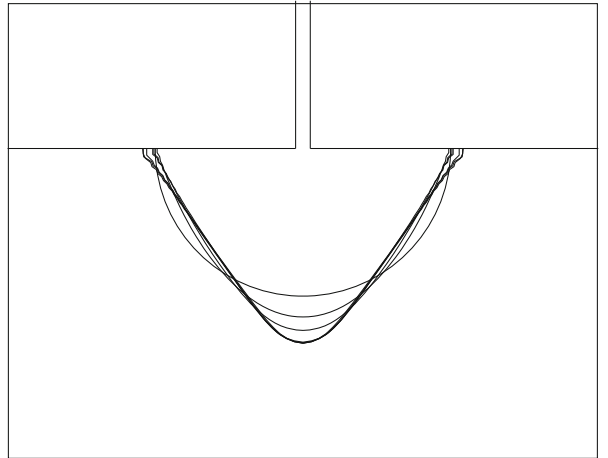

Fig. 14 Development of a stable shape after 30 iterations, for $E_{c} / E_{s}=0.1$ and $\gamma / \gamma_{0}=0.1$ with (Only every fifth iteration is illustrated.)

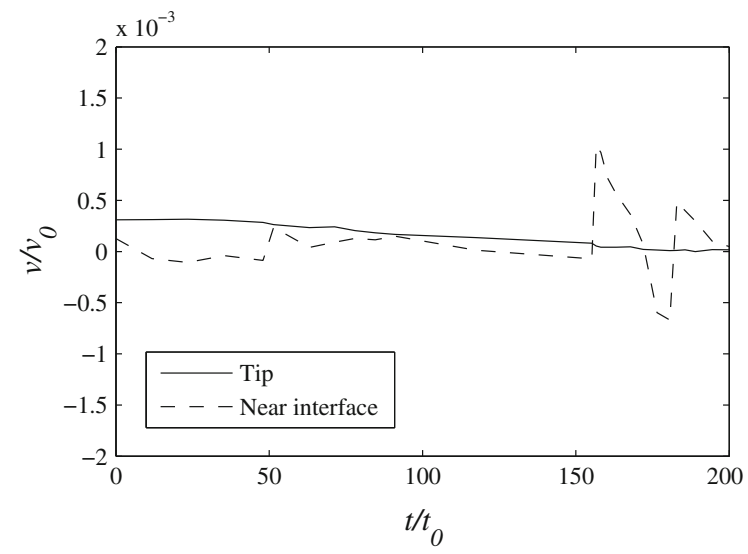

Fig. 15 Evolution rate at the bottom of the pit (tip) and near the interface for the case with a weaker coating $\left(E_{c} / E_{s}=0.1\right)$

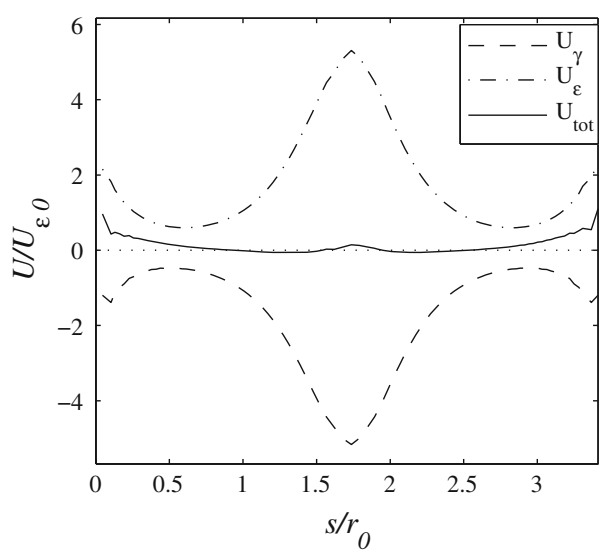

Fig. 16 Strain and surface energy density distributions along the pit surface, for increment number 25 , together with the sum of the energies substantially with stiffness ratio. For the three different cases, the strain energy density at the tip, $U_{\varepsilon, \text { tip }}$ is approximately equal to $2.4 \cdot 10^{4} U_{\varepsilon 0}, 450 U_{\varepsilon 0}$ and $20 U_{\varepsilon 0}$, respectively. Meaning that, the stiffer the coating is, the higher the stress concentration is at the pit bottom. This also means that risk for a corrosion attack is much larger for a substrate with a damaged stiff coating than with a weaker one. This is easily understood if one consider the pit and the crack in the coating together as a notch with a blunt tip, with the depth $a$ and the notch tip radius $r$. For a slender notch in a homogeneous material that is loaded perpendicular to the notch extension, the maximum stress, $\sigma_{\max }$, is found at the notch tip. According to Eq. (7) in Tada et al. (2000), $\sigma_{\max }$ can be calculated using the Mode I stress intensity factor, $K_{\mathrm{I}}$, for a corresponding sharp crack:

$\sigma_{\max }=2 K_{\mathrm{I}} \sqrt{\pi r}$.

For a sharp crack loaded by a nominal strain $\varepsilon_{0}$,

$K_{\mathrm{I}}=Y \bar{E}_{\mathrm{hom}} \varepsilon_{0} \sqrt{\pi a}$,

where $Y$ is a factor depending on geometry only, and for an edge crack $Y$ is approximately $1.12 . \bar{E}_{\text {hom }}$ represents Young's modulus for the homogeneous material.

Now consider the different bimaterial combinations for which $E_{c} / E_{s}$ equals $1 / 10,1$ and 10 , respectively. To estimate $K_{\mathrm{I}}$, the notch is approximated with a sharp crack in a homogenous material, i.e. Eq. (11) is used. With a weak coating $\left(E_{c} / E_{s}=1 / 10\right)$, the coating is disregarded so $\bar{E}_{\text {hom }}=\bar{E}_{s}$ and the crack length is chosen as the pit depth, i.e. $a=r$. In the case of a stiff coating $\left(E_{c} / E_{s}=10\right)$, the coating is assumed to be the homogeneous material $\bar{E}_{\mathrm{hom}}=\bar{E}_{c}$ and $a=100 \mathrm{r}$. With equal stiffness, we have $a=100 r$ and $\bar{E}_{\text {hom }}=$ $\bar{E}_{s}$.

An expression for the strain at the rounded notch tip, $\varepsilon_{\text {tip }}$, is found using the actual local stiffness $\bar{E}_{\text {local }}$ and not $\bar{E}_{\text {hom }}$, which was used for estimating $K_{\mathrm{I}}$. Together with Eqs. (10) and (11) we get:

$\varepsilon_{\text {tip }}=\frac{\sigma_{\text {max }}}{\bar{E}_{\text {local }}}=2 Y \frac{\bar{E}_{\text {hom }}}{\bar{E}_{\text {local }}} \varepsilon_{0} \sqrt{\pi a}$.

The strain energy density at the tip is then given by Eq. (4):

$U_{\varepsilon, \text { tip }}=\bar{E}_{\text {local }} \varepsilon_{\text {tip }}^{2} / 2=4 Y^{2} \frac{\bar{E}_{\text {hom }}^{2}}{2 \bar{E}_{\text {local }}} \varepsilon_{0}^{2} a / r$.

Hence, the relationship between $U_{\varepsilon, \text { tip }}$ and $U_{\varepsilon 0}$ can be written as:

$U_{\varepsilon, \text { tip }}=4 Y^{2} \frac{\bar{E}_{\text {hom }}^{2}}{\bar{E}_{\text {local }} \bar{E}_{s}} \frac{a}{r} U_{\varepsilon 0}$. 
In all cases, the notch tip is located the substrate and thus $\bar{E}_{\text {local }}=\bar{E}_{S}$. With $\bar{E}_{\text {hom }}$ and $a$ for the different cases as given above, we get $U_{\varepsilon, \text { tip }} \approx 5 U_{\varepsilon 0}$ for the weak coating, $U_{\varepsilon \text {,tip }} \approx 5 \cdot 10^{4} U_{\varepsilon 0}$ for the stiff one, and for the homogeneous case $U_{\varepsilon \text {,tip }} \approx 500 U_{\varepsilon 0}$. This coarse approximation gives $U_{\varepsilon \text {, tip }}$ of the same order of magnitude as those found in the simulations, even though the redistribution due to presence of the interface is not taken into account. The interface will instead strongly influence the shape evolution.

\section{Further remarks}

Jivkov (2004) has studied corrosion fatigue cracks that grow towards and through a bimaterial interface between two materials having different stiffness, using the same method and evolution law as presented by Jivkov and Ståhle (2002). It was found that cracks always will pass the interface regardless of stiffness ratio. In the case of entering the stiffer material from the weaker, the crack will retard, but not stop completely. During the passage, blunting of the crack tip and initiation of branching take place. When the crack leaves the interface, shape and speed are then regained. With a stiff-weak interface, the crack growth rate increases fast while approaching the interface, and after passage drops. In the present paper, the coating is already cracked and crack growth initiated in the substrate. Thus, the growth through the interface is not considered. However, the change of crack shape could be compared. To correspond to the conditions in Jivkov's investigation, we compare with a bimaterial with a medium tough interface (Type I). Only relatively low $\gamma$ values are relevant, since the flattening effect of the surface energy density is not considered by Jivkov. As earlier pointed out, the stresses are concentrated at the interface for both a weaker and a stiffer coating, cf. Fig. 13 where $U_{\varepsilon}$ along the initial pit surface is depicted. This means that with a sufficiently low $\gamma$, branching of the type shown in Fig. 10a is likely to develop in the substrate regardless if the coating is stiffer or weaker than the substrate. If only the branch growing into the substrate will continue to grow in the long run, as was observed by Jivkov, cannot be inferred from our results.

The deepening and branching pits with blunt crack tips can only be transformed into sharp cracks if the crack growth mechanism is changed, e.g. if some microstructural features have to be taken into account and the continuum approach is limited. The numerical method has some limitations regarding mesh sensitivity. For a denser mesh, the same shapes but with slower growth rates are found, and vice versa. The results are also influenced by the size of the chosen maximum evolution step, $l_{\max }$. For larger steps, the pit is mainly enlarged and the different shapes have not the possibility to develop. However, with small enough $l_{\max }$, the different characteristic shapes are obtained. The influence of a very large range of interface energy factors is studied. This has to be seen in the light of that only one loading case is considered, and that the value of $\gamma$ is specific for each substrate-environment systems.

The different types of crack (or pit) shapes found in this study seem to correlate well with some microscopic findings reported in the literature, cf. (Sopok et al. 2005a,b; Underwood et al. 2004, 2007). The material removal in these cases is perhaps not only due to classical corrosion, but also erosion, wear and thermal degradation. Nevertheless, the chemical potential is essential for probably all material transformation and transportation mechanisms, which motivated us to conduct this study.

\section{Conclusions}

In the present study, the evolution of stress corrosion is investigated that is initiated at the interface between a coated substrate material and an inert coating that contains through-thickness cracks. A remote load is applied parallel to the interface. A method to simulate anodic stress corrosion cracking due to material dissolution is presented. The evolution of an initial pit is treated as a two-dimensional moving boundary problem, where the dissolution rate is assumed to be a function of strain and surface energy densities along the surface.

The influence of interface toughness is investigated by using different geometric restrictions for surface evolution in a near interface zone: -A weak interface render sharp interface cracks. -Branching occurs for the medium resistance case. One branch follows the interface, whereas the other one grows inwards into the substrate material. Both types of branches have blunted tips. -For a tough interface, the initial pit deepens and narrows without easily forming a crack like shape.

The influence of the surface energy density is studied by varying a parameter that governs the impact of 
curvature on the surface evolution. -It is found that for high values of this parameter the evolution of cracks was efficiently held back. -For low values, the strain energy density dominates the phenomenon and thus promotes crack growth.

Different stiffness ratios between coating and substrate are also studied. -For both a stiffer and a weaker coating, the bi-material wedge acts as stress concentrator, thus resulting in that the different characteristic shapes can develop depending on interface energy density of solid-fluid interface and interface toughness. -For the case with a weaker coating, an equilibrium shape was achieved for a certain $\gamma$ value. The pit evolved slowly into a V-shaped notch with a blunt tip and an opening angle of approximately $70^{\circ}$.

The numerical method has some limitations regarding mesh sensitivity and the results are also influenced by the size of a maximum step. However, the different types of crack or pit shapes found in this study correlate with microscopic findings reported in the literature.

This pilot study indicates that including energy considerations may contribute to the understanding of evolution of dissolution driven stress corrosion cracks in the vicinity of a bi-material interface.

Acknowledgments C. Bjerkén was financially supported by the Swedish Research Council, (VR 50562401-02,50562402$02)$. This support is greatly acknowledged. M. Ortiz would like to acknowledge the support of the United States Army Research office through the award: W911NF-06-0421 Mod/Amend\#: P0001.

Open Access This article is distributed under the terms of the Creative Commons Attribution Noncommercial License which permits any noncommercial use, distribution, and reproduction in any medium, provided the original author(s) and source are credited.

\section{References}

ABAQUS User's Manual, Version 6.7 (2007) ABAQUS, Inc., Dassault Systé mes
Asaro RJ, Tiller WA (1972) Interface morphology development during stress corrosion cracking: part I. Via surface diffusion. Metall Trans 3:1789-1796

Bjerkén C (2010) The influence of biaxial loading on branching of a dissolution driven stress corrosion crack. Eng Fract Mech. doi:10.1016/j.engfracmech.2010.03.026

Dundurs J (1969) Edge-bonded dissimilar orthogonal elastic wedges. J Appl Mech 36:650-652

Grinfeld M (1986) Instability of the separation boundary between a non-hydrostatically stressed elastic body and a melt. Sov Phys Dokl 31:831-834

Jivkov AP, Ståhle P (2002) Strain-driven corrosion crack growth a pilot study of intergranular stress corrosion cracking. Eng Fract Mech 69:2095-2111

Jivkov AP (2004) Fatigue corrosion crack extension across the interface of an elastic bi-material. Eng Fract Mech 71:11191133

Kim K-S, Hurtado JA, Tan H (1999) Evolution of a surfaceroughness spectrum caused by stress in nanometer-scale chemical etching. Phys Rev 83:3872-3875

Shewchuk JR (2002) Delaunay refinement algorithms for triangular mesh generation. Comp Geom Theor Appl 22:21-74

Sopok S, Rickard C, Dunn S (2005) Thermal-chemical-mechanical gun bore erosion of an advanced artillery system part one: theories and mechanisms. Wear 258:659-670

Sopok S, Rickard C, Dunn S (2005) Thermal-chemical-mechanical gun bore erosion of an advanced artillery system part one: modelling and predictions. Wear 258:671-683

Srolovitz DJ (1989) On the stability of surfaces of stressed solids. Acta Metall 37:621-625

Ståhle P, Bjerkén C, Jivkov AP (2007) On dissolution driven crack growth. Int J Solids Struct 44:1880-1890

Tada H, Paris PC, Irwin GR (2000) Stress analysis of cracks handbook, 3rd edn. ASME Press, New York, 613

Underwood JH, Witherell MD, Sopok S, McNeil JC, Mulligan CP, Vigilante GN (2004) Thermomechanical modeling of transient thermal damage in cannon bore materials. Wear 257:992-998

Underwood JH, Vigilante GN, Mulligan CP (2007) Review of thermo-mechanical cracking and wear mechanisms in large caliber guns. Wear 263:1616-1621 